\title{
Efficient Multimodality Volume Fusion Using Graphics Hardware
}

\author{
Helen Hong ${ }^{1}$, Juhee $\mathrm{Bae}^{2}$, Heewon $\mathrm{Kye}^{2}$, and Yeong Gil Shin ${ }^{2}$ \\ ${ }^{1}$ School of Computer Science and Engineering, \\ BK21: Information Technology, Seoul National University \\ hlhong@cse.snu.ac.kr \\ ${ }^{2}$ School of Computer Science and Engineering, Seoul National University, \\ San 56-1 Shinlim 9-dong, Kwanak-gu, Seoul 151-742, Korea \\ \{jhbay, kuei, yshin\}@cglab.snu.ac.kr
}

\begin{abstract}
We propose a novel technique of multimodality volume fusion using graphics hardware that solves the depth cueing problem with less time consumption. Our method consists of three steps. First, it takes two volumes and generates sample planes orthogonal to the viewing direction following 3D texture mapping volume rendering. Second, it composites textured slices each from different modalities with several compositing operations. Third, alpha blending for all the slices is performed. For the efficient volume fusion, a pixel program is written in HLSL(High Level Shader Language). Experimental results show that our hardware-accelerated method distinguishes the depth of overlapping region of the volume and renders them much faster than conventional ones on software.
\end{abstract}

\section{Introduction}

In clinical medicine, several different modalities such as Positron Emission Tomography (PET), Computed Tomography (CT), and Magnetic Resonance Imaging (MRI) are useful for radiologists and surgeons to help diagnosis support and treatment planning. These modalities give complementary information when they are shown simultaneously. Thus many medical applications require visual output generated from multimodality volumes rather than only one volume.

Several methods have been proposed to combine multiple volumes obtained from multimodality imaging. Cai and Sakas [1] proposed three different intermixing approaches. The shortcoming of the method was a lack of precise depth cueing among the multiple volumes, even though they tried to use Z-buffer value. Jacq and Roux [2] presented a multi-volume rendering focused on material classification which applied different material percentages and merging rules at each sampling point. Zuiderveld et al. [3] described how to cache calculated voxel properties using sized hash table that contribute to the final result. Previous researches have performed multimodality volume fusion on software. However, the software-based multimodality fusion has a limitation in depth presentation and processing time. In this paper, we propose an efficient hardwareaccelerated solution to combine multimodality volumes at interactive rates. 


\section{Multimodality Volume Fusion}

Fig. 1 shows the pipeline of our method for multimodality volume fusion. We assume reference and float volume datasets have the same orientation by setting rigid transformation that would be sufficient for the registration of the datasets [4].

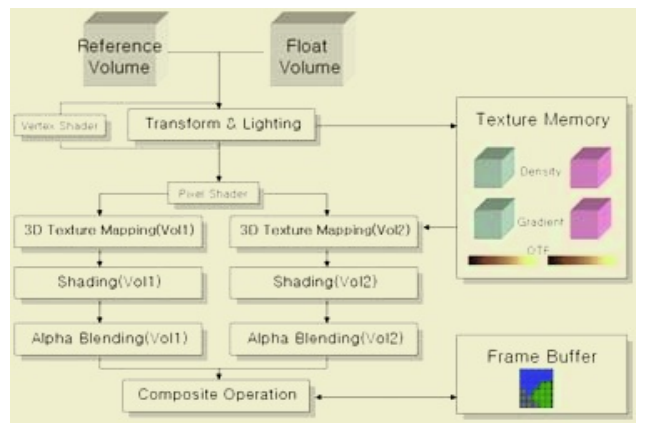

Fig. 1. The pipeline of multimodality volume fusion

\subsection{Depth Compositing of the Volume}

Since multiple volumes have different size and orientation, it needs correct depth composition and orientation. Fig. 2 shows different ways to combine two slices from each volume. In Fig. 2(a), it is required to set the orders of each slice of the volumes which is a troublesome work. We combine each texture with same texture coordinates from different volumes before blending as shown in Fig. 2(b). Two voxels (ultimately texels) referred to the same texture coordinates are combined by the compositing operations and then they are mapped to the same slice.

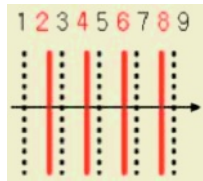

(a)

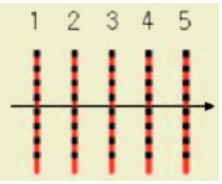

(b)

Fig. 2. A comparison of blending textures (black dotted line: slice of reference volume, red solid line: slice of float volume) (a): alpha blending in slice orders (b): combine textures before alpha blending

\subsection{Compositing Operations for Volume Fusion}

Several compositing operations are experimented to find an appropriate combining method; some are referred from the previous compositing researches [7][8]. 
The results of combined color and opacity of over, XOR, plus and weighted addition operation are shown from Eq. (1) to Eq. (4), respectively.

$$
\begin{aligned}
& C=\frac{\alpha_{A} \cdot C_{A}+\left(1-\alpha_{A}\right) \alpha_{B} \cdot C_{B}}{\alpha_{A}+\left(1-\alpha_{A}\right) \cdot \alpha_{B}} \\
& \alpha=\alpha_{A}+\left(1-\alpha_{A}\right) \cdot \alpha_{B} \\
& C=C_{A}+C_{B} \\
& \alpha=\alpha_{A}+\alpha_{B} \\
& C=\frac{\left(1-\alpha_{B}\right) \cdot \alpha_{A} \cdot C_{A}+\left(1-\alpha_{A}\right) \alpha_{B} \cdot C_{B}}{\left(1-\alpha_{B}\right) \cdot \alpha_{A}+\left(1-\alpha_{A}\right) \cdot \alpha_{B}} \\
& \alpha=\left(1-\alpha_{B}\right) \cdot \alpha_{A}+\left(1-\alpha_{A}\right) \cdot \alpha_{B} \\
& \begin{array}{l}
C=\frac{\text { weight } \cdot \alpha_{A} \cdot C_{A}+(1-\text { weight }) \cdot \alpha_{B} \cdot C_{B}}{\text { weight } \cdot \alpha_{A}+(1-\text { weight }) \cdot \alpha_{B}} \\
\alpha=\text { weight } \cdot \alpha_{A}+(1-\text { weight }) \cdot \alpha_{B}
\end{array} \\
& (0 \leq \text { weight } \leq 1)
\end{aligned}
$$

where $\mathrm{C}$ indicates color, $\alpha$ indicates alpha, $\mathrm{A}$ refers to the first volume, and $\mathrm{B}$ to the second volume.

\subsection{Weighted Opacity for Surface Extraction}

To see a float volume through a reference volume, we consider weight to the opacity as shown in Eq. (5). The weight value tells whether the pixel is a part of the surface or a part of the homogeneous region by gradient magnitude. In the preprocessing step, gradient magnitude is used as normal for shading. Here, we use gradient magnitude as a numerical divergence which implies that the surface has higher value while homogeneous part has a lower value. During fusion process, we let the homogeneous area to be transparent so that we could see through the reference volume by eliminating the opacity value of the homogeneous area between reference and float volume of the overlapping area.

$$
\text { weight }= \begin{cases}0 & \text { if }(|\nabla|<c) \\ 1 & \text { else }\end{cases}
$$

\section{Experimental Results}

All our implementation and tests have been performed on an Intel Pentium IV PC containing 2.53 GHz CPU with ATI Radeon X800 256 MB RAM. Our method

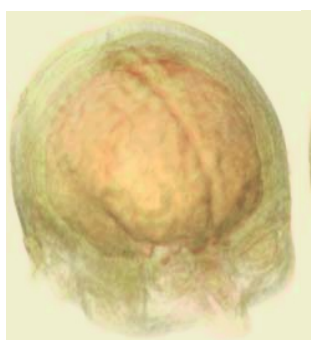

(a) (b)

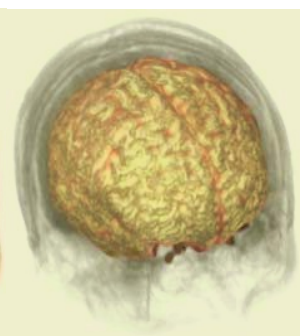

(c) (d)

Fig. 3. The results of compositing operations at opacity 8. (a) over (b) XOR(exclusive OR) (c)weight addition (d) plus operation 
has been applied to the brain of $256 \times 256 \times 96$ MR images and $128 \times 128 \times 40$ PET images with 2 bytes-intensity. The performance of our method is evaluated with the aspects of visual inspection and processing time.

Fig. 3 displays the results using several compositing operations. Fig. 3(a) and Fig. 3(b) show the result of over operation and XOR(exclusive OR). Fig. 3(c) using weight addition displays rather a dark but manifest result compared to others. Fig. 3(d) represents a result of plus operation to be mainly white because the colors are simply added which induces overflow.

The average of the processing time of the fusion volume supported by software is $3703 \mathrm{msec}$ while fusion on hardware is $625 \mathrm{msec}$, respectively. We gained about 5 to 6 times of speed enhancement on rendering time.

\section{Conclusion}

We have developed an efficient multimodality volume fusion method using graphics hardware. Our method presents several approaches of combining two different volume datasets into a singular image representation. MR and PET images of the brain have been used for the performance evaluation with the aspects of visual inspection and processing time. Our method shows the exact depth of each volume and the realistic views with interactive rate in comparison with the software-based multimodality volume fusion. Distinguishable and fast result of the proposed method can be successfully utilized in medical diagnosis.

\section{References}

1. Cai W., Sakas G., Data Intermixing and Multi-volume Rendering, Computer Graphics Forum (1999), 18(3): 359-368.

2. Jaeq, J., Roux, C., A Direct Multi-volumes Rendering Methods Aiming at Comparison of 3D Images and Methods, IEEE Trans. On Information Technology in Biomedicine (1997) 1(1):30-43.

3. Zuiderveld, K.J., Viergever, M.A., Multi-modal Volume Visualization using Objectoriented methods, in Proc. IEEE/ACM Volume Visualization '94 Symposium (1994) 59-66.

4. Hong, H., Shin, Y., Intensity-based registration and combined visualization of multimodal brain images for noninvasive epilepsy surgery planning, Proc. of SPIE Medical Imaging (2003). 